\title{
Methane emissions from cattle: estimates from short-term measurements using a Green Feed system compared with measurements obtained using respiration chambers or sulphur hexafluoride tracer
}

Article

Accepted Version

Hammond, K., Humphries, D., Crompton, L., Green, C. and Reynolds, C. (2015) Methane emissions from cattle: estimates from short-term measurements using a Green Feed system compared with measurements obtained using respiration chambers or sulphur hexafluoride tracer. Animal Feed Science and Technology, 203. pp. 41-52. ISSN 0377-8401 doi: https://doi.org/10.1016/j.anifeedsci.2015.02.008 Available at https://centaur.reading.ac.uk/39710/

It is advisable to refer to the publisher's version if you intend to cite from the work. See Guidance on citing.

Published version at: http://www.sciencedirect.com/science/article/pii/S0377840115000590

To link to this article DOI: http://dx.doi.org/10.1016/j.anifeedsci.2015.02.008

Publisher: Elsevier

All outputs in CentAUR are protected by Intellectual Property Rights law, including copyright law. Copyright and IPR is retained by the creators or other copyright holders. Terms and conditions for use of this material are defined in 
the End User Agreement.

www.reading.ac.uk/centaur

\section{CentAUR}

Central Archive at the University of Reading

Reading's research outputs online 
7 Methane emissions from cattle: estimates from short-term measurements using a GreenFeed

8 system compared with measurements obtained using respiration chambers or sulphur

9 hexafluoride tracer

10 K.J. Hammond, D.J. Humphries, L.A. Crompton, C. Green, and C.K. Reynolds*

11

12 School of Agriculture, Policy and Development, Centre for Dairy Research, University of 13 Reading, PO Box 237, Earley Gate, Reading RG 6AR, United Kingdom

14

15

16

17

18

19

20

21

22

23 * Corresponding author. Tel: +44 118 3784684; Fax +44 1183786595

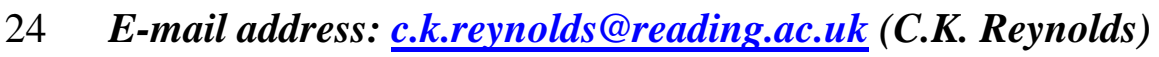




\section{Abstract}

The GreenFeed (GF) system (C-Lock Inc., Rapid City, USA) is used to estimate total daily methane emissions of individual cattle using short-term measurements obtained over several days. Our objective was to compare measurements of methane emission by growing cattle obtained using the GF system with measurements using respiration chambers (RC) or sulphur hexafluoride tracer $\left(\mathrm{SF}_{6}\right)$. It was hypothesised that estimates of methane emission for individual animals and treatments would be similar for GF compared to $\mathrm{RC}$ or $\mathrm{SF}_{6}$ techniques. In experiment 1 , maize or grass silage-based diets were fed to four growing Holstein heifers, whilst for experiment 2, four different heifers were fed four haylage treatments. Both experiments were a 4 x 4 Latin square design with 33 d periods. GreenFeed measurements of methane emission were obtained over $7 \mathrm{~d}$ (days 22-28) and compared to subsequent RC measurements over 4 d (days 29-33). For experiment 3, 12 growing heifers rotationally grazed three swards for $26 \mathrm{~d}$, with simultaneous $\mathrm{GF}$ and $\mathrm{SF}_{6}$ measurements over two $4 \mathrm{~d}$ measurement periods (days 15-19 and days 22-26). Overall methane emissions (g/d and $\mathrm{g} / \mathrm{kg}$ dry matter intake [DMI]) measured using GF in experiments 1 (198 and 26.6, respectively) and 2 (208 and 27.8, respectively) were similar to averages obtained using RC (218 and 28.3, respectively for experiment 1; and 209 and 27.7, respectively, for experiment 2); but there was poor concordance between the two methods ( 0.1043 for experiments 1 and 2 combined). Overall, methane emissions measured using $\mathrm{SF}_{6}$ were higher $(\mathrm{P}<0.001)$ than GF during grazing (186 vs. $164 \mathrm{~g} / \mathrm{d})$, but there was significant $(\mathrm{P}<0.01)$ concordance between the two methods (0.6017). There were fewer methane measurements by GF under grazing conditions in experiment $3(1.60 / \mathrm{d})$ compared to indoor measurements in experiments 1 $(2.11 / d)$ and $2(2.34 / d)$. Significant treatment effects on methane emission measured using $\mathrm{RC}$ and $\mathrm{SF}_{6}$ were not evident for GF measurements, and the ranking for treatments and individual animals differed using the GF system. We conclude that under our conditions of 
50 use the GF system was unable to detect significant treatment and individual animal 51 differences in methane emissions that were identified using both $\mathrm{RC}$ and $\mathrm{SF}_{6}$ techniques, in

52 part due to limited numbers and timing of measurements obtained. Our data suggest that 53 successful use of the GF system is reliant on the number and timing of measurements 54 obtained relative to diurnal patterns of methane emission.

55

56 Keywords: Dairy cattle, methane, respiration chamber, $\mathrm{SF}_{6}$, GreenFeed

57

58 Abbreviations: CI, confidence interval; DM(I), dry matter (intake); GF, GreenFeed; LW, live 59 weight; LWG, LW gain; NDIR, non-dispersive infrared; RC, respiration chambers; RFID, 60 radio frequency identification; $\mathrm{SF}_{6}$, sulphur hexafluoride tracer 


\section{Introduction}

Accurate and robust measurements of methane emissions from individual animals are required for national inventories and assessment of mitigation strategies. There are a number of methods for determining methane emissions from ruminants, including respiration chambers $(\mathrm{RC})$ and sulphur hexafluoride tracer $\left(\mathrm{SF}_{6}\right)$ techniques. Precise measurements of methane emission can be obtained by housing animals in RC, which allow direct measurement of total methane emission. However, RC are relatively expensive, have a limited throughput, and are disruptive to normal behaviour as animal by environment interactions that occur within grassland ecosystems are prevented. Respiration chambers are impractical for simulating grazing applications, and if the diet offered in the RC is fresh forage, then diet selection is limited, and eating patterns are likely to be determined by the feeding regime.

The $\mathrm{SF}_{6}$ technique (Zimmerman, 1993; Johnson et al., 1994) can be used to make estimations of eructated and expired methane emissions from animals which can select their diet in a manner representative of farmed livestock (e.g. grazing). However, evaluations have challenged the precision of the $\mathrm{SF}_{6}$ technique for estimating methane emissions (Vlaming et al., 2007; Pinares-Patiño and Clark, 2008; Pinares-Patiño et al., 2011), with greater betweenanimal variation compared to RC (Hammond et al., 2009). The $\mathrm{SF}_{6}$ technique has also provided variable estimates of methane emission from animals on different herbages that have not been corroborated by RC measurements (e.g. Hammond et al., 2011; Waghorn et al., 2002; Sun et al., 2011 and 2012). Halter and collection canisters placed on the animal for methane estimates can interfere with grazing (Pinares-Patiño et al., 2008), especially with young animals, and a lower than predicted feed dry matter intake (DMI) will overestimate methane yields (g/kg DMI). Rumen $\mathrm{SF}_{6}$ boluses must also be administered, and frequent 
animal handling is needed, all of which can be disruptive to normal behaviour, and is relatively labour intensive.

In 2010, the commercial GreenFeed (GF) system (C-Lock Inc., Rapid City, South Dakota, USA) was introduced as a static short-term measurement device that measures methane emission from individual cattle, and uses head position sensors in combination with decision rules to assess the validity of measures obtained. The animal is free to move about and voluntarily enters a hood where a feed supplement (i.e. a reward for visiting the GF unit) is delivered. Measurements of methane emission by GF are typically over short (3-7 min) periods, several times within a day, over several days. The system is programmed using CLock Inc. software to control timing of feed availability and thus, encourage animals to distribute their voluntary GF visitation and hence methane measurements over a $24 \mathrm{~h}$ period so that ultimately a $24 \mathrm{~h}$ individual methane emission profile can be extrapolated from several days of short-term measurements. Cattle are typically not handled during GF operation and one GF unit can be used for numerous animals, with manufacturer recommendations of 15-20 animals/unit when grazing and 20-25 animals/unit if housed in free stalls. Because the GF system is relatively new, little is known about its operation, precision, accuracy, and the extent to which animal interaction with GF affects methane measurements.

The objectives of the present study were addressed across three experiments that included measurements of methane emission from individual growing dairy cattle using a single GF unit. Our objectives were to compare measurements of methane emission by growing dairy cattle obtained using GF with measurements using RC (experiments 1 and 2) and $\mathrm{SF}_{6}$ (experiment 3). It was hypothesised that estimates of methane emission for individual animals and treatments would be similar for $\mathrm{GF}$ and $\mathrm{RC}$ or $\mathrm{SF}_{6}$ techniques.

\section{Materials and methods}


110 Three experiments were used in this study whereby growing dairy cattle were fed a

111 variety of diets and methane emission was measured using $\mathrm{GF}, \mathrm{RC}$ and/or $\mathrm{SF}_{6}$ techniques.

112 Measurements in all experiments were individual DMI and methane production (g/d),

113 calculated methane yield ( $\mathrm{g} / \mathrm{kg} \mathrm{DMI})$, and frequency of GF visitation (i.e. methane

114 measurement frequency). All procedures used were approved and monitored under the UK

115 Home Office Animals (Scientific Procedures) Act 1986.

116

$117 \quad 2.1$ Experiments

$118 \quad 2.1 .1$ Experiment 1

119 Four Holstein Friesian dairy heifers aged 14 months with an initial live weight (LW)

120 of $317 \pm 20 \mathrm{~kg}$ were fed once daily $(10: 00 \mathrm{~h})$ either maize or grass silage diets supplemented

121 with or without an extruded linseed product (Lintec; $26 \%$ fat) at $6 \%$ of ration DM ( $n=4$

122 animals/treatment).

123 Experiment 1 was a 4 x 4 Latin square design with each period $33 \mathrm{~d}$ in duration,

124 commencing with $21 \mathrm{~d}$ adaptation where access to GF was allowed, and GF data used for

125 analysis was obtained during $7 \mathrm{~d}$ (days 22-28), after which animals were confined to RC for

126 measurement of methane emission over 4 d (days 29-33).

127 Feed was offered to achieve target daily LW gains (LWG) of $0.75 \mathrm{~kg}$. Feed intakes

128 were measured on a daily basis using an electronic Calan Broadbent individual feeding

129 system (American Calan Inc., Northwood, New Hampshire, USA) with feed refusals

130 collected once daily before morning feeding. Animals were loose-housed and bedded on

131 wood shavings with rubber mats and had ad libitum access to water.

\subsubsection{Experiment 2}

Four Holstein Friesian dairy heifers, aged 14 months with an initial LW of $339 \pm 16$

$134 \mathrm{~kg}$ were fed twice daily (10:00 and 16:00 $\mathrm{h}$ in equal amounts), one of four conserved forage 
135 (haylage) treatments of ryegrass, clover, trefoil and flowers ( $n=4$ animals/treatment). Further

136 details of these treatments are given in Hammond et al. (2014). Similar to experiment 1,

137 experiment 2 was a 4 x 4 Latin Square design with 33 d periods, with animals fed and housed

138 in a similar manner as detailed for experiment 1.

$139 \quad$ 2.1.3 Experiment 3

140 Twelve Holstein Friesian dairy heifers aged eight months, with a starting LW of 230

$141 \pm 6 \mathrm{~kg}$, grazed the same treatments used to make haylage in experiment 2 (ryegrass, clover

142 and flowers; $n=12$ animals/treatment; Hammond et al., 2014). Heifers rotationally strip

143 grazed each sward treatment for $26 \mathrm{~d}$ in a sequence of flowers, clover, then ryegrass. Each 26

$144 \mathrm{~d}$ period commenced with $14 \mathrm{~d}$ adaptation where GF access was allowed, with simultaneous

145 GF and $\mathrm{SF}_{6}$ data obtained over two 4-d measurement periods (days 15-19 and days 22-26).

146 Dry matter intake was estimated using a rising plate meter (Farmworks Precision

147 Farming Systems, Feilding, New Zealand) by taking 20 sward height readings before and

148 after each days grazing period. Sward DM yield estimations were calibrated every second day

149 by taking $5 \times 0.5 \mathrm{~m}^{2}$ quadrat cuts of the sward at a target post-grazing height of $6 \mathrm{~cm}$ and 150 oven drying $\left(100^{\circ} \mathrm{C}\right)$ the sample to give sward $\mathrm{DM}$ yield per $\mathrm{m}^{2}$ which was applied to each 151 sward height measurement.

\subsection{Methane measurements}

$154 \quad 2.2 .1$ GreenFeed

155 The GF system measured methane emission using sensors that identified the animal 156 and its head position within a sampling hood, air flow, and methane and carbon dioxide 157 concentrations in exhaust air. GreenFeed operation was initiated when the animal placed its 158 head inside the hood. A radio frequency identification (RFID) reader identified the animal's 159 ear tag and GF sampling was activated when the animals head (located by an infrared sensor) 
160 was in the correct location within the hood, and it was deemed that sufficient time had 161 elapsed since the previous methane measurement for that animal.

Animal head position was critical for successful measurements as the animal is free to

163 move its head in and out of the hood and thus only data captured with uninterrupted

164 measurements was retained for statistical analysis. Position of the animals head within the

165 hood was monitored using sensors to ensure complete breath collection. Adequate animal 166 head position resulted in the dispensing of feed pellets which were used for enticement and

167 encouraged the animal to maintain a suitable head position for accurate measurements.

168 Pellets were dispensed from a hopper above the GF using a computer controlled rotating cup

169 dispenser.

170 Animals were able to use the GF unit at any time, provided it was not in use by another animal, however, this did not necessarily generate a measurement of methane. A 'visit' is defined as a visit that results in a methane measurement. Thus, a 'visit' is only considered when a certain time has elapsed between visits (as dictated by the user) and a food reward is dropped, generating a methane measurement for that animal.

The concentration of the gas emitted by the animal was calculated using background gas concentration, the differential concentration of gas during the animal's time in the GF hood, and the calibration coefficient for concentration. The calibration coefficient was based on nitrogen, carbon dioxide and methane gases used to calculate the response of the sensors.

179 The GF analysers were zeroed and calibrated weekly using zero baseline gas (oxygen-free 180 nitrogen) and a span gas mixture nitrogen containing 5000 ppm carbon dioxide and 1000 ppm methane (BOC Ltd., Manchester, UK). This was to account for any drift in the calibration of

182 the analysers, which was found to be negligible. A known amount of propane or carbon 183 dioxide was released near where the animal's nose would be when feeding to check recovery 
184 of expired gases when the physical location of the GF unit changed. There was no recovery correction required in the current study.

To measure gas production (mass per unit of time) an extractor fan was used to draw air past the animal's head into an exhaust pipe and airflow rate was measured. Airflow rate was multiplied by the increase in gas concentration when the animals head was in the hood. The duration the animals head was in the GF hood was recorded thus giving the time interval for calculation of mass per unit of time. The concentrations of methane and carbon dioxide gases were measured by non-dispersive infrared (NDIR) sensors, and an air filter was used to filter and remove any fine particulate material from the air that was subsampled to the sensors to prevent damage. The air filter was changed every two weeks. Data from GF was available real-time using mobile phone communication through a web-based data management system provided by C-Lock Inc.

For all experiments, the GF was programmed using C-Lock Inc. software to deliver a maximum of five rotations of a feed dispensing cup, delivering approximately $55 \mathrm{~g}$ of pellet (as fed) per rotation, with intervals of $45 \mathrm{sec}$ between each rotation, so that $275 \mathrm{~g}$ of pellet was delivered during each visit. A maximum of four visits per day ( $24 \mathrm{~h})$ was allowed, with a minimum of $4 \mathrm{~h}$ required between visits. Therefore, if an animal attempted to use the GF less than $4 \mathrm{~h}$ from the previous visit pellets would not be dispensed. Commercial calf pellets (Rearer18 Nuts, Wynnstay Group PLC, UK) were used for GF enticement and had a chemical composition (g/kg DM) of ash, 85.1; oil, 46.5; acid detergent fibre, 174; neutral detergent fibre, 289; starch, 259; water soluble carbohydrate, 91.3; nitrogen, 27.3, crude protein, 171; and gross energy (MJ/kg), 18.1. In all experiments total daily feed allocations included $1 \mathrm{~kg}$ of expected pellet DM provided by the GF unit.

The GF unit was set up indoors for experiments 1 and 2 at one end of animal housing, with gates positioned to restrict access to one animal at a time. Barn ventilation was used to 
maintain ambient concentrations of methane in background air. For experiment 3, the GF unit was located outdoors under an awning at a point central to the experimental paddocks. The

211 GF was located next to the only available water trough to encourage visitation, and fences

212 and tracks were established to provide continuous access from grazed paddocks.

\section{$213 \quad$ 2.2.2 Respiration chambers}

214 Details of the RC and measurements of methane emission are given by Reynolds et al.

215 (2001) and Cammell et al. (1986). For measurements of gaseous emissions, two open-circuit

216 RC were used (internal volume approximately $21 \mathrm{~m}^{3}$ ), with air-locks enabling access for

217 faecal and urine collection (Cammell et al., 1986). An integrative sample of ambient and RC

218 exhaust air was analysed at 4-min intervals, and every $4 \mathrm{~h}$ there was a switch to calibration

219 gases (oxygen-free nitrogen and nitrogen carrier with 20.5\%, $3000 \mathrm{ppm}$, and $200 \mathrm{ppm}$ oxygen, carbon dioxide, and methane, respectively) to provide gas analyses with variation coefficients of $5 \%$ or less.

\subsubsection{Sulphur hexafluoride}

Experiment 3 used the $\mathrm{SF}_{6}$ technique, as detailed previously by Hammond et al. (2014). Two weeks prior to experiment 3 commencing, heifers were each dosed by mouth with a $\mathrm{SF}_{6}$ permeation tube (supplied by AFBI, Hillsborough, Northern Ireland, UK) into the rumen. The $\mathrm{SF}_{6}$ gas release rates from the permeation tubes $(5.176 \pm 0.248 \mathrm{mg} / \mathrm{d})$ were measured prior to dosing by oven incubation at $39^{\circ} \mathrm{C}$ and weighing twice weekly for six weeks. Daily methane emissions from heifers were estimated from analysis of air collected from around the nose and mouth over a $24 \mathrm{~h}$ period into a pre-evacuated PVC canister which was suspended under the neck. Based on recommendations given by Berndt et al. (2014), air was sampled using a crimped stainless steel capillary 0.004 " ID $10 \mathrm{~cm}$ tube, with a flow rate between 0.45 to $0.55 \mathrm{ml} / \mathrm{min}$. Canisters had a volume of approximately $2.3 \mathrm{~L}$ and a precollection vacuum of $90 \mathrm{kPa}$. Canisters were changed once daily at the same time each 
morning and were rejected if vacuum post-collection was $>75$ or $<50 \mathrm{kPa}$. A background air sample was also obtained daily from the paddock adjacent to that being grazed. Samples from canisters were analysed daily in our laboratory using gas chromatography to determine methane and $\mathrm{SF}_{6}$ concentrations as described by Muñoz et al. (2012).

\subsection{Data and statistical analyses}

Data from GF and RC during periods 1 and 2 of experiment 1 were excluded from the analyses because the methane concentration of the calibration gas used for the GF unit was too low. Thus, comparisons for experiment 1 were restricted to periods 3 and $4(n=8)$ which meant treatment effects were not tested due to the limited observations obtained with the Latin Square design experiment. Each animal and period emissions data generated by GF was averaged over $7 \mathrm{~d}$, whereas $\mathrm{RC}$ data was averaged over $4 \mathrm{~d}$, with data expressed on a per min basis over $24 \mathrm{~h}$ and as a daily average $(\mathrm{g} / \mathrm{h}$ and $\mathrm{g} / \mathrm{d})$.

For experiment 2 , data from all four animals and periods were analysed statistically ( $n$

$248=16$ ) using the Mixed Models Procedures of SAS (2011) for random effects of animal and

249 fixed effects of period and treatment. Like experiment 1, each animal and period emissions data generated by GF was averaged over $7 \mathrm{~d}$, whereas RC data was averaged over $4 \mathrm{~d}$ in experiment 2, with data expressed on a per min basis over $24 \mathrm{~h}$ and as a daily average $(\mathrm{g} / \mathrm{h}$ and $\mathrm{g} / \mathrm{d})$.

Experiment 3 provided methane data for 12 heifers grazing three fresh forage treatments for two 4-d methane measurement periods and two treatment periods (May to July and August to October; Hammond et al., 2014). Analysis of methane emission data were undertaken on daily averages across $4 \mathrm{~d}$ of measurements for both $\mathrm{GF}$ and $\mathrm{SF}_{6}$ techniques (obtained simultaneously) for individual animals during each measurement period. Twelve heifers were used for the first grazing rotation of ryegrass, clover, and flowers, and also for 
the second rotation of flowers. However, for the second rotation on ryegrass and clover, two

260 animals were removed because there was insufficient sward cover. Therefore, a total of 136

261 GF and $\mathrm{SF}_{6}$ individual animal average emission rate observations were analysed using Mixed

262 Models Procedure of SAS (2011) for effects of forage treatment and treatment period (1 or

263 2), with 4-d measurement period within forage treatment period as a repeated effect within

264 heifers (Hammond et al., 2014). When significant effects occurred, means of forage mixtures

265 (clover and flowers) were differentiated from ryegrass control using Dunnett's adjusted mean

266 comparisons.

Differences in methane emission (g/h, g/d and g/kg DMI) between techniques (GF $v s$.

$\mathrm{RC}$ and $\mathrm{GF} v s . \mathrm{SF}_{6}$ ) across all experiments were tested using Lin's Concordance Correlation

269 Coefficient analysis (Lin et al., 1998) in GenStat (2010) and the Univariate Procedure of SAS

270 (2011) to determine if the difference between the two methods for each experiment was

271 different from zero.

Within each experiment, the Least Squares Mean option of the GLM procedure (SAS, 2011) was used to rank individual animals according to their methane emission $(\mathrm{g} / \mathrm{d}$ and $\mathrm{g} / \mathrm{kg}$ DMI) for each measurement technique using animal as a fixed effect. In addition, the GLM procedure was used to regress GF measurements against $\mathrm{RC}$ or $\mathrm{SF}_{6}$ measurements $(\mathrm{g} / \mathrm{d})$.

\section{3. Results}

$278 \quad 3.1$ General observations

\subsubsection{Experiment 1}

As stated previously, experiment 1 included data from four animals with $n=8$

281 observations (only two periods out of a possible four were used). Dry matter intake during

282 GF and RC measurements was similar (Table 1). Average methane production (g/d) and yield 283 (g/kg DMI), determined using either GF or RC techniques, was similar for individual animals 
284 (198 vs. $215 \mathrm{~g} / \mathrm{d}$, and 26.6 vs. $28.3 \mathrm{~g} / \mathrm{kg}$ DMI, for GF vs. RC techniques, respectively) (Table

285 1). Individual animals had a similar methane output regardless of measurement technique 286 used, however methane data (g/d and g/kg DMI) generated by GF and RC techniques ranked 287 heifers differently in numerical order from high to low methane output (data not shown).

$288 \quad 3.1 .2$ Experiment 2

289 There were four heifers used in experiment 2 with 16 observations (all four periods 290 included). Heifers had a similar DMI during GF and RC measurements (Table 1). Average 291 daily methane production $(\mathrm{g} / \mathrm{d})$ and yield $(\mathrm{g} / \mathrm{kg} \mathrm{DMI})$ did not differ with measurement 292 technique for individual animals (208 vs. $209 \mathrm{~g} / \mathrm{d}$, and 27.8 vs. $27.7 \mathrm{~g} / \mathrm{kg}$ DMI, for GF vs. RC 293 techniques, respectively; Table 1). For both GF and RC methods, animals were significantly $294(\mathrm{P}=0.05)$ different to each other in their methane production but not methane yield. Both GF and RC techniques ranked animals in numerical order, from low to high, the same for methane production, but not for methane yield (data not shown).

\subsubsection{Experiment 3}

Experiment 3 used 12 heifers and had 136 observations. Approximately $88 \%$ of $\mathrm{SF}_{6}$ canisters were accepted (478 measurements out of a possible 544), with $12 \%$ of measurements unsuccessful due to sampling tube blockages, broken collection tubes, or displacement of canisters from the heifer. Both $\mathrm{GF}$ and $\mathrm{SF}_{6}$ techniques were used simultaneously so DMI was the same with measurement technique. Daily methane

303 production determined by GF for individual heifers was lower $(\mathrm{P}<0.001)$ than $\mathrm{SF}_{6}(164$ vs.

$304186 \mathrm{~g} / \mathrm{d}$, respectively; Table 3$)$. For both $\mathrm{GF}$ and $\mathrm{SF}_{6}$ methods, heifers were significantly $(\mathrm{P}=$ 305 0.05) different to each other in their methane production $(\mathrm{g} / \mathrm{d})$, and the ranking of animals, 306 from low to high methane production, was different for the two techniques. 
3.2 Technique comparisons

311

312

313

314

\subsubsection{GreenFeed vs. respiration chamber}

Combining data from experiments 1 and 2, Lin's Concordance Correlation Coefficient between GF and RC, when used to measure methane production and yield of individual heifers, was 0.1043 and 0.058 , respectively, with a non-significant $(\mathrm{P}>0.50)$ association between the two techniques, based on the $95 \%$ confidence interval (CI) (Fig 1). There were diurnal patterns of methane erucation over a $24 \mathrm{~h}$ period for animals in both experiments 1 and 2, measured using GF and in RC (Fig 2). Emissions ranged from about 4 $\mathrm{g} / \mathrm{h}$ immediately before their morning feeding to a maximum of about $15 \mathrm{~g} / \mathrm{h}$ after feeding, on both silage and haylage diets. The increase in methane production after 10:00 $\mathrm{h}$ in experiment 1 relates to once daily feeding, whereas the increases just after 10:00 and 16:00 h represent the twice daily feeding regime. Based on all methane measurements, compared to the GF data, there was less variability with the $\mathrm{RC}$ emission measurements (g/d) from both experiments, in part because measurements for GF were much less frequent and fewer in number than for RC (Fig 2).

\section{Insert Fig 1 here}

Insert Fig 2 here

\subsubsection{GreenFeed vs. sulphur hexafluoride}

Lin's Concordance Correlation Coefficient between GF and $\mathrm{SF}_{6}$ techniques, used to measure methane production from individual heifers of experiment 3 , was 0.602 , with a significant $(\mathrm{P}<0.01)$ association between the two techniques, based on the 95\% CI (Fig 3). 
Insert Fig 3 here

335

\subsection{GreenFeed for detecting dietary treatment effects}

In experiment 2, DMI and methane production during $\mathrm{RC}$ measurements was affected by haylage type $(\mathrm{P}=0.045$ and $\mathrm{P}=0.025$, respectively), but this was not evident for $\mathrm{GF}$ measurements (Table 2). When methane was expressed in terms of DMI (yield, g/kg DMI), $\mathrm{RC}$ detected significant differences $(\mathrm{P}=0.020)$ between haylages, but $\mathrm{GF}$ did not. There was no consistency in the relative difference between measurement techniques with dietary treatment. Relative to RC, GF underestimated $15 \%$ of methane yield when heifers were fed a ryegrass diet, compared to an overestimation of $12 \%$ for heifers on a flower diet (Table 2).

For heifers of experiment 3 , methane production $(\mathrm{g} / \mathrm{d})$ differed significantly with both GF and $\mathrm{SF}_{6}$ techniques $(\mathrm{P}=0.019$ and $\mathrm{P}<0.001$, respectively) for all three forage treatments. However, the ranking of mean estimates for the different forages differed with technique (Table 2). When methane was expressed in terms of DMI (methane yield), noting that the techniques estimated methane simultaneously, the ranking of treatment means was not the same for $\mathrm{GF}(\mathrm{P}=0.080$; flowers $>$ clover $=$ ryegrass $)$ and $\mathrm{SF}_{6}$ techniques $(\mathrm{P}=0.002$; clover $=$ ryegrass $>$ flowers). For two out of three dietary treatments fed, GF underestimated methane yield relative to $\mathrm{SF}_{6}$ by up to $18 \%$ (Table 2).

Insert Table 2 here

\subsection{GreenFeed visitation}

During the $14 \mathrm{~d}$ of GF measurements in experiment 1 , there were a total of 118 visits to the GF unit, averaging 2.11 visits/d. For the $28 \mathrm{~d}$ measurement period in experiment 2, total GF visitation was 262, averaging 2.34 visits/d. During the $48 \mathrm{~d}$ of measurements for experiment 3, heifers visited the GF unit 880 times, averaging 1.60 visits/d (Table 3). The 
average duration (min:sec) of GF measurements for experiments 1, 2, and 3 were 04:44, 04:43, and 04:58, respectively.

Figure 4 shows the pattern of visits to the GF, according to hour of the day. For all experiments, animals frequented the GF most often between 07:00 and 08:00 h, and between 13:00 and 14:00 h, with fewer visits in early morning hours (between 01:00 and 06:00 h). GreenFeed measurements were prevented if another animal was already using the unit, when animals were yarded for other experimental activities such as $\mathrm{SF}_{6}$ canister changes, and during the allocation of new grazing. The type of diet offered affected GF visitation for 0.001) visits overall to the GF when on the ryegrass (219) and clover (229) paddocks, compared to the flower (432) paddock.

370

Insert Table 3 here

Insert Fig 4 here

\section{Discussion}

\subsection{Comparison of measurement techniques}

\subsubsection{GreenFeed vs. respiration chamber}

Based on the concordance analysis for methane emission from heifers of experiments

3781 and 2, GF and RC techniques had a poor agreement, yet average methane emission overall was similar for the two techniques. It is difficult to interpret these conflicting results; however the large amount of variation about the line of equality (Fig 1) is a likely explanation

381 for overall methane means being similar between techniques (Table 1) but having low concordance correlation. The lack of concordance between methods is in part attributable to the relatively small number of short-term measurements obtained by GF on each day of 
measurement. The concept behind the GF system is that although it is unknown what an animal is eructating when not visiting the GF, the accumulation of data over $24 \mathrm{~h}$ can provide a representative pattern (Fig 2). Thus, the GF technique relies on the animal visiting the unit at different times during the day to characterise the daily pattern of methane emission over a number of days. In contrast, RC measurements in this study were based on integrated measurements every 4 min over four consecutive days.

The inability of GF to detect changes in methane production due to treatment or animal effects compared to $\mathrm{RC}$ (and $\mathrm{SF}_{6}$ ) is not unexpected given the methodology the technique employs. Enteric methane production from ruminants typically exhibits a diurnal pattern related to feeding and meal consumption, with methane emission rate varying by as much as five-fold over the course of a day (Crompton et al., 2010). Peak enteric methane production occurs approximately 120 and $60 \mathrm{~min}$ after the morning and afternoon feeds, respectively, for a lactating dairy cow fed ad libitum twice daily (Crompton et al., 2010). Frequent or continuous measurements over a $24 \mathrm{~h}$ period using $\mathrm{RC}$ or $\mathrm{SF}_{6}$ account for any diurnal variation in methane production, but intermittent short-term measurements may vary significantly depending on when those measurements are taken during the day.

There was a greater range in absolute emissions for both measurement techniques with experiment 2 data compared to experiment 1 that was associated with greater differences in DMI and methane production. However, when emissions were expressed per unit of feed intake (g/kg DMI), relationships between GF and RC measurements were still weak and variation was large, especially for the GF measurements. The variable relationship suggests that the differences in methane emission due to treatments and animal variation measured by the RC are not correlated with differences measured by GF. In other words, ranking of the animals according to methane production and yield differed between the two techniques, despite substantial differences being observed. The absence of a significant correlation 
409 between GF and RC measurements for individual animal observations (Fig 1) casts doubt on

410 the capability of GF to distinguish (and rank) individual animals under the conditions with

411 which GF was used in our experiments. With the exception of daily methane production in

412 experiment 2, GF and RC ranked heifers differently in their methane emission. Daily mean

413 methane production varied from about 160 to $270 \mathrm{~g} / \mathrm{d}$ measured in RC, and although GF also

414 recorded a similar range, the range was for different heifers on different diets (data not 415 shown).

416 It is possible that the alogrithms used by the GF system for the calculation of methane 417 output, or the timing of visits relative to daily patterns of methane emission, may account for 418 the discrepencies observed between GF and RC data. GreenFeed calculations are based on 419 differences in the concentration of the air exhaled and eructed by the animal, less background 420 air concentrations measured pre- and post-feeding. The GF is able to differentiate emissions 421 of methane in exhaled air above background, so exhaled air is included in the emission 422 calculation. The calculations are reliant on erucation events taking place within the 423 measurement period, and the alogrithms may need to be modified to increase accuracy and 424 reduce variation. For the animals and diets used in our study, more GF measurements were 425 needed over a longer period, and at more frequent intervals, to better represent the diurnal 426 pattern of methane emissions over $24 \mathrm{~h}$. Increased animal visitation to the GF may require 427 longer periods of measurement (more days), more visits per day (and thus greater feed 428 consumption), or the use of an alternative 'bait' (Hegarty, 2013). In addition to this, it has 429 been estimated that $1-2 \%$ of methane is voided as flatus (Murray et al., 1976), and it will 430 contribute to methane emissions (Ellis et al., 2008) measured in RC. These considerations for 431 GF measurements are also pertinent to other on-farm breath analysis techniques (e.g. 432 Garnsworthy et al., 2012). 


\subsubsection{GreenFeed vs. sulphur hexafluoride}

GreenFeed and $\mathrm{SF}_{6}$ techniques had moderate concordance (agreement), in part due to the greater number of observations compared. However, overall methane emissions determined using GF were significantly lower than those measured using the $\mathrm{SF}_{6}$ technique. Differences between GF and $\mathrm{SF}_{6}$ techniques are likely due to the duration of methane measurements obtained for each animal. $\mathrm{The}_{\mathrm{SF}}$ technique is based on integrative sampling with a sampling duration of nearly $1440 \mathrm{~min} / \mathrm{d}(100 \%$ of $24 \mathrm{~h})$. In comparison, the GF unit is designed to take intermittent samples, and based on the average of the three experiments presented here, sampling duration (5 min/visit at 2 visits/animal/d) was only $10 \mathrm{~min} / \mathrm{d}(0.7 \%$ of $24 \mathrm{~h})$.

All tracers have weaknesses (Shipley and Clark, 1972) and the variation associated with $\mathrm{SF}_{6}$ estimates may be in part a consequence of factors affecting the technique itself (Deighton et al., 2013, 2014a and 2014b), or alternatively the variation might be real. Recent work has found that successful use of the $\mathrm{SF}_{6}$ technique to detect differences in enteric methane emissions due to diets or between animal species may be confounded by diet or genetic effects on body temperature (Deighton et al., 2014b). In order to accurately determine methane emissions, it is necessary that gases are collected continuously at a constant rate for 24 h, however; it has been recently shown by Deighton et al. (2014a) that capillary tubes are unsuitable for use as flow restrictors to achieve this, causing a bias of up to $15.6 \%$ in calculated methane emissions. Deighton et al. (2014a) has since proposed a 'modified $\mathrm{SF}_{6}$ technique' which incorporates orifice plate flow restrictors for $24 \mathrm{~h}$ sample collection and has found technique error associated with $\mathrm{SF}_{6}$ release, sample collection and analysis to be reduced. 
Although all three techniques measured significant treatment effects on methane emissions, the ranking of these effects differed with measurement technique. Critically, both the $\mathrm{RC}$ and $\mathrm{SF}_{6}$ techniques found methane yield ( $\left.\mathrm{g} / \mathrm{kg} \mathrm{DMI}\right)$ to be the lowest in both experiments 2 and 3 for animals fed flowers compared to the other dietary treatments (Hammond et al., 2014). GreenFeed on the other hand, was unable to detect treatment effects on methane yield in experiment 2 , and ranked the treatments differently to $\mathrm{SF}_{6}$ for experiment 3. This in part reflects the variability of GF measurements attributable to the timing and limited number of short-term measurements obtained in the present experiments.

\subsection{GreenFeed visitation}

Although animals had few problems adapting to the GF and used it willingly, visits were less frequent than permitted, particularly for grazing animals in experiment 3 . The lack of GF visits from animals both while out grazing and during early morning, may have negatively biased methane production measured by GF (Fig 3). The low frequency of visits between 09:00 and 13:00 h (Fig 4) is likely to be when peak methane emissions occur in a once daily feeding system, as can be seen from the rise in methane production $(\mathrm{g} / \mathrm{h})$ in Fig 4. Thus, the infrequent daily measurements made by the GF system in experiment 1 is a likely explanation for numerically lower methane emissions from the GF compared to RC. In experiment 2, the GF pattern of visitation was better distributed over the course of the day, although a weaker relationship occurred between the two techniques (Fig 2).

In all experiments, fewer visits occurred in the early morning hours, and a lack of methane data over this period may have affected the average estimate of daily emissions.

481 Every morning, heifers of experiment 3 were given a new allocation of feed at about 10:30 h after $\mathrm{SF}_{6}$ canisters were replaced. The allocation of new pasture is likely to have been responsible for the drop in GF visits between 09:00 and 13:00 h. During this period of time, 
methane emissions would have been at their highest, which must partly account for the $13 \%$ greater daily methane emissions determined by the $\mathrm{SF}_{6}$ technique, compared to the GF system. The lower visitation to the GF by grazing heifers is cause for concern, especially as the GF system relies on having enough daily measurements over the course of an 'average' day to estimate daily emissions. Further evaluations of the GF system should determine the number of days and measurements per day required for GF to provide accurate and precise measures of methane emissions.

It would appear that the number of visits to the GF is influenced by dietary treatment (and possibly level of feed intake), with more visits made when heifers were grazing flowers, compared to ryegrass and clover (experiment 3 ). For experiment 3 , this may have been attributable to the location of the GF relative to the paddocks, as well as the DM and nutrient content of the swards grazed. It would appear that less favourable diets may contribute to increased GF visitation when a favourable 'treatment' i.e. pelleted concentrates, is rewarded, and this may have consequences for methane estimates on different treatments (different numbers of samples and sampling times for each treatment). This is a concern for nutrition experiments that investigate effects of diet composition on methane emission if diet comparisons are affected by varying amounts of feed reward provided by GF.

One concern with the use of the GF under our conditions is the temporal distribution of GF visitation and the potential for bias in methane emission measurements by the GF system. This is because unlike both RC and SF6 techniques the methane measurement 504 obtained from each individual animal by the GF system is voluntary and thus not completely 505 independent. The use of the GF unit by each individual animal within the group, and therefore the temporal distribution of their methane measurement, is affected by their cohorts and environmental circumstances. The inclusion of a given animal in the GF unit causes the exclusion of all other animals within the group. Strictly speaking an individual animal is not 
509 totally independent as an experimental unit when the GF system is used and therefore to

510 achieve completely independent experimental replication for individual animals housed as

511 groups in pens or paddocks multiple GF units may be required.

512

\section{5. Conclusions}

514 Overall, the GF system provided an estimate of methane emission by growing dairy 515 cattle that was not different from RC measurements, but significantly lower than for $\mathrm{SF}_{6}$. 516 However, concordance analyses found no agreement between GF and RC, and only moderate 517 agreement with $\mathrm{SF}_{6}$. We conclude that as used in our experiments, the GF system was unable 518 to detect significant treatment and individual animal differences in methane emissions that 519 were identified using both $\mathrm{RC}$ and $\mathrm{SF}_{6}$ techniques. The successful use of the GF system is 520 reliant on the number and timing of measurements obtained relative to diurnal patterns of methane emission. Therefore, animal and diet type, intake level and appetite (e.g. ad libitum $v s$. restrictive feeding), total feed availability, accessibility of the GF unit relative to other

523 feeds and activities, as well as type, amount, and timing of feed used to elicit GF use all 524 affect GF visitation and thus measurements of methane emission using the GF system. 525 Multiple animals using a GF unit can alter the temporal distribution of measurements for individual animals and this potential bias should also be considered in designing future 527 experiments.

Further evaluation of GF is needed to determine how best to deploy the system to meet specific objectives, the number and timing of measurements required for specific measurement conditions, as well as the capacity of the GF to detect significant changes in methane emissions with individuals and treatments. We suggest that in addition to increased

532 frequency of daily GF visits future studies should include longer periods of measurement and 533 a greater number of animals per treatment than is required for RC studies. 


\section{Acknowledgements}

536 This study was funded by Defra, the Scottish Government, DARD, and the Welsh

537 Government as part of the UK's Agricultural GHG Research Platform project 538 (www.ghgplatform.org.uk). Contributions from the technical staff of CEDAR in the daily

539 routine of experiments and care of cows, as well as assistance from C-Lock Inc. with regards 540 to GreenFeed operation and use, is gratefully acknowledged.

\section{$542 \quad$ References}

543 Berndt, A., Boland, T.M., Deighton, M.H., Gere, J.I., Grainger, C., Hegarty, R.S., Iwaasa, 544 A.D., Koolaard, J.P., Lassey, K.R., Luo, D., Martin, R.J., Martin, C., Moate, P.J., Molano, 545 G., Pinares-Patiño, C.S., Ribaux, B.E., Swainson, N.M., Waghorn, G.W., Williams, S.R.O., 546 2014. Guidelines for use of sulphur hexafluoride $\left(\mathrm{SF}_{6}\right)$ tracer technique to measure enteric 547 methane emissions from ruminants. Pp 166. [M.G. Lambert, editor], New Zealand 548 Agricultural Greenhouse Gas Research Centre, New Zealand.

550 Cammell, S.B., Thomson, D.J., Beever, D.E., Haines, M.J., Dhanoa, M.S., Spooner, M.C., 1986. The efficiency of energy utilization in growing cattle consuming fresh perennial ryegrass (Lolium perenne cv. Melle) or white clover (Trifolium repens cv. Blanca). Br. J.

553 Nutr. 55, 669-680.

555 Crompton, L.A., Mills, J.A.N., Reynolds, C.K. and France, J., 2010. Fluctuations in methane emission in response to feeding pattern in lactating dairy cows. In. Modelling Nutrient 
558 Faverdin and N. Friggens, editors]. Wageningen, the Netherlands: Wageningen Academic 559 Publishers.

560

561 Deighton, M.H., O’Loughlin, B.M., Williams, S.R.O., Moate, P.J., Kennedy, E., Boland,

562 T.M., Eckard, R.J., 2013. Declining sulphur hexafluoride permeability of

563 polytetrafluoroethylene membranes causes overestimation of calculated ruminant methane

564 emissions using the tracer technique. Anim. Feed Sci. Technol. 183, 86-95.

565

566 Deighton, M.H., Williams, S.R.O., Hannah, M.C., Eckard, R.J., Boland, T.M., Wales, W.J.,

567 Moate, P.J., 2014a. A modified sulphur hexafluoride tracer technique enables accurate 568 determination of enteric methane emissions from ruminants. Anim. Feed Sci. Technol. 197, $569 \quad 47-63$.

570

571 Deighton, M.H., Williams, S.R.O., Lassey, K.R., Hannah, M.C., Boland, T.M., Eckard, R.J., 572 Moate, P.J., 2014b. Temperature, but not submersion or orientation, influences the rate of 573 sulphur hexafluoride release from permeation tubes used for estimation of ruminant methane emissions. Anim. Feed Sci. Technol. 194, 71-80.

575

576 Ellis, J.L., Dijkstra, J., Kebreab, E., Bannink, A., Odongo, N.E., McBride, B.W., France, J., 577 2008. Aspects of rumen microbiology central to mechanistic modelling of methane 578 production in cattle. J. Agric. Sci. 146, 213-233.

579

580 Garnsworthy, P.C., Craigon, J., Hernandez-Medrano, J.H., Saunders, N., 2012. On-farm 581 methane measurements during milking correlate with total methane production by individual 582 dairy cows. J. Dairy Sci. 95, 3166-3180. 
584 Hammond, K.J., Hoskin, S.O., Burke, J.L., Waghorn, G.C., Koolaard, J.P., Muetzel, S., 2011.

585 Effects of feeding fresh white clover (Trifolium repens) or perennial ryegrass (Lolium 586 perenne) on enteric methane emissions from sheep. Anim. Feed Sci. Technol. 166-167, 398587404.

588

589 Hammond, K.J., Humphries, D.J., Westbury, D.B., Thompson, A., Crompton, L.A., Kirton, 590 P., Green, C., Reynolds, C.K., 2014. The inclusion of forage mixtures in the diet of growing 591 dairy heifers: Impacts on digestion, energy utilisation, and methane emissions. Agric. Eco. 592 Environ. 197, 88-95.

593

594 Hammond, K.J., Muetzel, S., Waghorn, G.C., Pinares-Patiño, C.S., Burke, J.L., Hoskin, S.O., 595 2009. The variation in methane emissions from sheep and cattle is not explained by the 596 chemical composition of ryegrass. Proc. N. Z. Soc. Anim. Prod. 69, 174-178.

597

598 Hegarty, R.S., 2013. Applicability of short-term emission measurements for on-farm 599 quantification of enteric methane. Anim. 7:s2, 401-408.

600

601 Johnson, K.A., Huyler, M., Westberg, H., Lamb, B., Zimmerman, P., 1994. Measurement of 602 methane emissions from ruminant livestock using a $\mathrm{SF}_{6}$ tracer technique. Environ. Sci. 603 Technol. 28, 359-362.

604

605 Lin, L., Torbeck, L.D., 1998. Coefficient of accuracy and concordance correlation 606 coefficient: new statistics for methods comparison. J. Pharm. Sci. Technol. 52, 55-59. 
608 Muñoz, C., Yan, T., Wills, D.A., Murray, S., Gordon, A.W., 2012. Comparison of the sulfur 609 hexafluoride tracer and respiration chamber techniques for estimating methane emissions and

610 correction for rectum methane output from dairy cows. J. Dairy Sci. 95, 3139-3148.

611

612 Murray, R.M., Bryant, A.M., Leng, R.A., 1976. Rates of production of methane in the rumen 613 and large intestine of sheep. Brit. J. Nutr. 36, 1-14.

614

615 Pinares-Patiño, C.S., Lassey, K.R., Martin, R.J., Molano, G., Fernandez, M., MacLean, S., 616 Sandoval, E., Luo, D., Clark, H., 2011. Assessment of the sulphur hexafluoride $\left(\mathrm{SF}_{6}\right)$ tracer 617 technique using respiration chambers for estimation of methane emissions from sheep. Anim. 618 Feed Sci. Technol. 166-167, 201-209.

619

620 Pinares-Patiño, C.S., Clark, H., 2008. Reliability of the sulfur hexafluoride tracer technique 621 for methane emission measurement from individual animals: an overview. Aust. J. Exp. 622 Agric. 48, 223-229.

623

624 Pinares-Patiño, C.S., Machmuller, A., Molano, G., Smith, A., Vlaming, J.B., Clark, H., 2008. $625 \mathrm{The}_{\mathrm{SF}}$ tracer technique for measurements of methane emission from cattle - effect of tracer 626 permeation rate. Can. J. Anim. Sci. 88, 309-320.

627

628 Reynolds, C.K., Cammell, S.B., Humphries, D.J., Beever, D.E., Sutton, J.D., Newbold, J.R., 629 2001. Effects of postrumen starch infusion on milk production and energy metabolism in 630 dairy cows. J. Dairy Sci. 84, 2250-2259. 
632 Shipley, R.A., Clark, R.E., 1972. Tracer Methods for In Vivo Kinetics: Theory and 633 Applications. Academic Press, New York.

634

635 Sun, X.Z., Hoskin, S.O., Muetzel, S., Molano, G., Clark, H., 2011. Effect of chicory 636 (Cichorium intybus) and perennial ryegrass (Lolium perenne) on methane emissions in vitro 637 and from sheep. Anim. Feed Sci. Technol. 166-167, 391-397.

638

639 Sun, X.Z., Hoskin, S.O., Zhang, G.G., Molano, G., Muetzel, S., Pinares-Patiño, C.S., Clark, 640 H., Pacheco, D., 2012. Sheep fed forage chicory (Cichorium intybus) or perennial ryegrass 641 (Lolium perenne) have similar methane emissions. Anim. Feed Sci. Technol. 172, 217-225.

642

643 Vlaming, J.B., Brookes, I.M., Hoskin, S.O., Pinares-Patiño, C.S., Clark, H., 2007. The 644 possible influence of intra-ruminal sulphur hexafluoride release rates on calculated methane 645 emissions from cattle. Can. J. Anim. Sci. 87, 269-275.

646

647 Waghorn, G.C., Tavendale, M.H., Woodfield, D.R., 2002. Methanogenesis from forages fed 648 to sheep. Proc. N. Z. Grassland Assoc. 64, 167-171.

649

650 Zimmerman, P.R., 1993. System for measuring metabolic gas emissions from animals. U.S 651 Patent 5265618, U.S. Patent and Trademark Office, November 30, 1993. 
652 Table 1 Dry matter intake (DMI; kg/d), methane production $(\mathrm{g} / \mathrm{d})$, and methane yield $(\mathrm{g} / \mathrm{kg}$

653 DMI) from growing dairy cattle within three different experiments using GreenFeed (GF),

654 respiration chamber $(\mathrm{RC})$ and sulphur hexafluoride tracer $\left(\mathrm{SF}_{6}\right)$ techniques.

\begin{tabular}{|c|c|c|c|c|c|c|}
\hline & $\begin{array}{l}\text { Experiment } \\
1^{\mathrm{a}}\end{array}$ & $\pm \mathrm{SD}$ & $\begin{array}{l}\text { Experiment } \\
2^{\mathrm{a}}\end{array}$ & $\pm \mathrm{SD}$ & $\begin{array}{l}\text { Experiment } \\
3^{\mathrm{b}}\end{array}$ & $\pm \mathrm{SD}$ \\
\hline \multicolumn{7}{|l|}{ DMI, kg/d } \\
\hline GF & 7.62 & 0.81 & 7.60 & 0.81 & 9.15 & 2.67 \\
\hline $\mathrm{RC}^{\mathrm{a}}$ or $\mathrm{SF}_{6}^{\mathrm{b}}$ & 7.66 & 0.59 & 7.54 & 0.94 & 9.15 & 2.67 \\
\hline$n$ & 8 & & 16 & & 136 & \\
\hline SEM & 0.132 & & 0.182 & & N/A & \\
\hline $\mathrm{P}$ & 0.799 & & 0.747 & & N/A & \\
\hline \multicolumn{7}{|l|}{ Methane, g/d } \\
\hline GF & 198 & 20.4 & 208 & 31.5 & 164 & 51.0 \\
\hline $\mathrm{RC}^{\mathrm{a}}$ or $\mathrm{SF}_{6}^{\mathrm{b}}$ & 215 & 22.3 & 209 & 30.9 & 186 & 57.3 \\
\hline$n$ & 8 & & 16 & & 136 & \\
\hline SEM & 9.230 & & 10.59 & & 2.900 & \\
\hline $\mathrm{P}$ & 0.170 & & 0.940 & & $<0.001$ & \\
\hline \multicolumn{7}{|c|}{ Methane, g/kg DMI } \\
\hline GF & 26.6 & 2.80 & 27.8 & 5.62 & 18.8 & 6.94 \\
\hline $\mathrm{RC}^{\mathrm{a}}$ or $\mathrm{SF}_{6}^{\mathrm{b}}$ & 28.3 & 3.01 & 27.7 & 1.81 & 21.5 & 7.60 \\
\hline$n$ & 8 & & 16 & & 136 & \\
\hline SEM & 1.365 & & 1.459 & & 0.349 & \\
\hline $\mathrm{P}$ & 0.255 & & 0.933 & & $<0.001$ & \\
\hline
\end{tabular}

${ }^{\mathrm{a}}$ Experiments 1 and 2 used RC for measurement of methane from dairy heifers.

${ }^{\mathrm{b}}$ Experiment 3 used $\mathrm{SF}_{6}$ for estimate of methane from grazing dairy heifers.

${ }^{c}$ DMI was measured using Calan gates for individual animals in experiments 1 and 2, however for experiment 3, DMI was estimated by pre- and post-herbage mass (hence same DMI for animals where both GF and $\mathrm{SF}_{6}$ were used simultaneously). 
Table 2 The difference in methane emission between GreenFeed $(\mathrm{GF})$, respiration chamber (RC), and sulphur hexafluoride tracer $\left(\mathrm{SF}_{6}\right)$ techniques with dairy heifers fed different dietary treatments.

\begin{tabular}{|c|c|c|c|c|c|c|c|c|c|}
\hline Experiments & $n$ & \multicolumn{2}{|c|}{$\begin{array}{l}\text { Dry matter intake } \\
(\mathrm{DMI}), \mathrm{kg} / \mathrm{d}^{\mathrm{c}}\end{array}$} & \multicolumn{2}{|c|}{$\begin{array}{c}\text { Methane production, } \\
\mathrm{g} / \mathrm{d}\end{array}$} & \multicolumn{4}{|c|}{$\begin{array}{l}\text { Methane yield, } \\
\text { g/kg DMI }\end{array}$} \\
\hline \multicolumn{10}{|c|}{ Experiment $2^{a}$} \\
\hline Ryegrass & 4 & 8.28 & 8.13 & 196 & 230 & 24.1 & 28.4 & -4.32 & -15 \\
\hline Clover & 4 & $6.86^{\mathrm{d}}$ & $7.10^{\mathrm{b}}$ & 202 & $200^{c}$ & 29.5 & 28.1 & 1.40 & 5 \\
\hline Trefoil & 4 & 7.93 & 7.51 & 226 & 218 & 28.9 & 29.2 & -0.32 & -1 \\
\hline P (haylage) & & 0.180 & 0.045 & 0.515 & 0.025 & 0.521 & 0.020 & 0.298 & \\
\hline \multicolumn{10}{|c|}{ Experiment $3^{b}$} \\
\hline Ryegrass & 44 & 10.0 & 10.0 & 175 & 204 & 17.3 & 21.8 & -3.38 & -16 \\
\hline Clover & 44 & $8.69^{a}$ & $8.69^{\mathrm{a}}$ & 166 & 202 & 18.5 & 23.0 & -4.24 & -18 \\
\hline Flowers & 48 & $8.78^{\mathrm{b}}$ & $8.78^{\mathrm{b}}$ & $159^{\mathrm{b}}$ & $159^{\mathrm{a}}$ & $19.7^{\mathrm{c}}$ & $19.5^{\mathrm{c}}$ & 0.48 & 2 \\
\hline
\end{tabular}

For each parameter, different letters indicate significant differences from the ryegrass control according to Dunnetts test $\left({ }^{\mathrm{a}} \mathrm{P}<0.001,{ }^{\mathrm{b}} \mathrm{P}<0.01\right.$, $\left.{ }^{\mathrm{c}} \mathrm{P}<0.05,{ }^{\mathrm{d}} \mathrm{P}<0.10\right)$.

${ }^{\text {a }}$ Experiment 2 used RC for measurement of methane from dairy heifers

${ }^{\mathrm{b}}$ Experiment 3 used $\mathrm{SF}_{6}$ for estimation of methane from grazing dairy heifers

${ }^{c}$ DMI was measured using calan gates for individual animals in experiment 2 , however for experiment 3 , DMI was estimated by pre- and postherbage mass (hence same DMI for animals in experiment 3 with measurement technique)

${ }^{\mathrm{d}}$ Difference is generated using GF value less corresponding $\mathrm{RC}$ or $\mathrm{SF}_{6}$ value 
659 Table 3 Animal visitation to the GreenFeed (GF) unit across three different experiments

660

\begin{tabular}{ccc}
\hline $\begin{array}{c}\text { Number of } \\
\text { measurement days }\end{array}$ & $\begin{array}{c}\text { Total number of } \\
\text { GF visits } \\
\text { (methane } \\
\text { measurements) }\end{array}$ & $\begin{array}{c}\text { Total number of GF visit } \\
\text { (methane measurements) } \\
\text { per animal per day }\end{array}$ \\
\hline
\end{tabular}

\section{Experiment 1}

Total

14

120

2.11

SD

SEM

0.49

0.17

\section{Experiment 2}

Ryegrass

76

2.71

Clover

60

2.14

Trefoil

68

2.43

Flowers

58

2.07

Total

28

262

2.34

SD

SEM

0.26

P (haylage)

0.425

\section{Experiment 3*}

Ryegrass

219

Clover

229

1.24

Flowers

432

$1.30^{\mathrm{d}}$

Total

48

880

$2.26^{\mathrm{a}}$

1.60

1.09

SEM

0.07

$\mathrm{P}$ (forage)

$<0.001$

* For experiment 3, different letters indicate significant differences from ryegrass control according to Dunnetts test $\left({ }^{\mathrm{a}} \mathrm{P}<0.001,{ }^{\mathrm{d}} \mathrm{P}<0.10\right)$ 


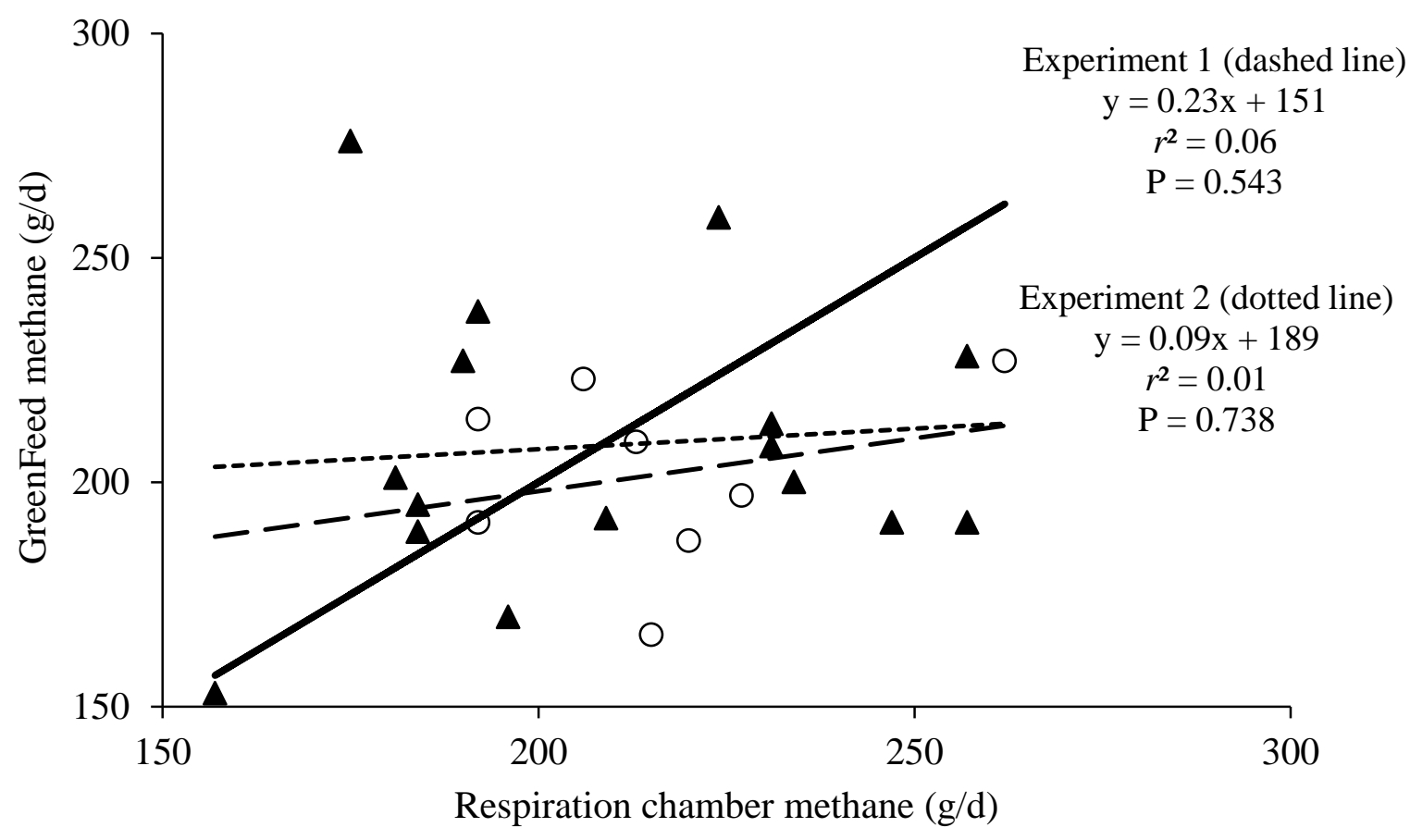

663 Fig 1. Relationships between methane production (g/d), determined using GreenFeed (GF)

664 and respiration chamber (RC) techniques, of individual dairy heifers in experiments 1 (open

665 circle symbol; $n=8$ ) and 2 (closed triangle symbol; $n=16$ ). Solid line indicates y $=\mathrm{x}$. Lin's

666 Concordance value for both experiments combined $=0.1043$. 


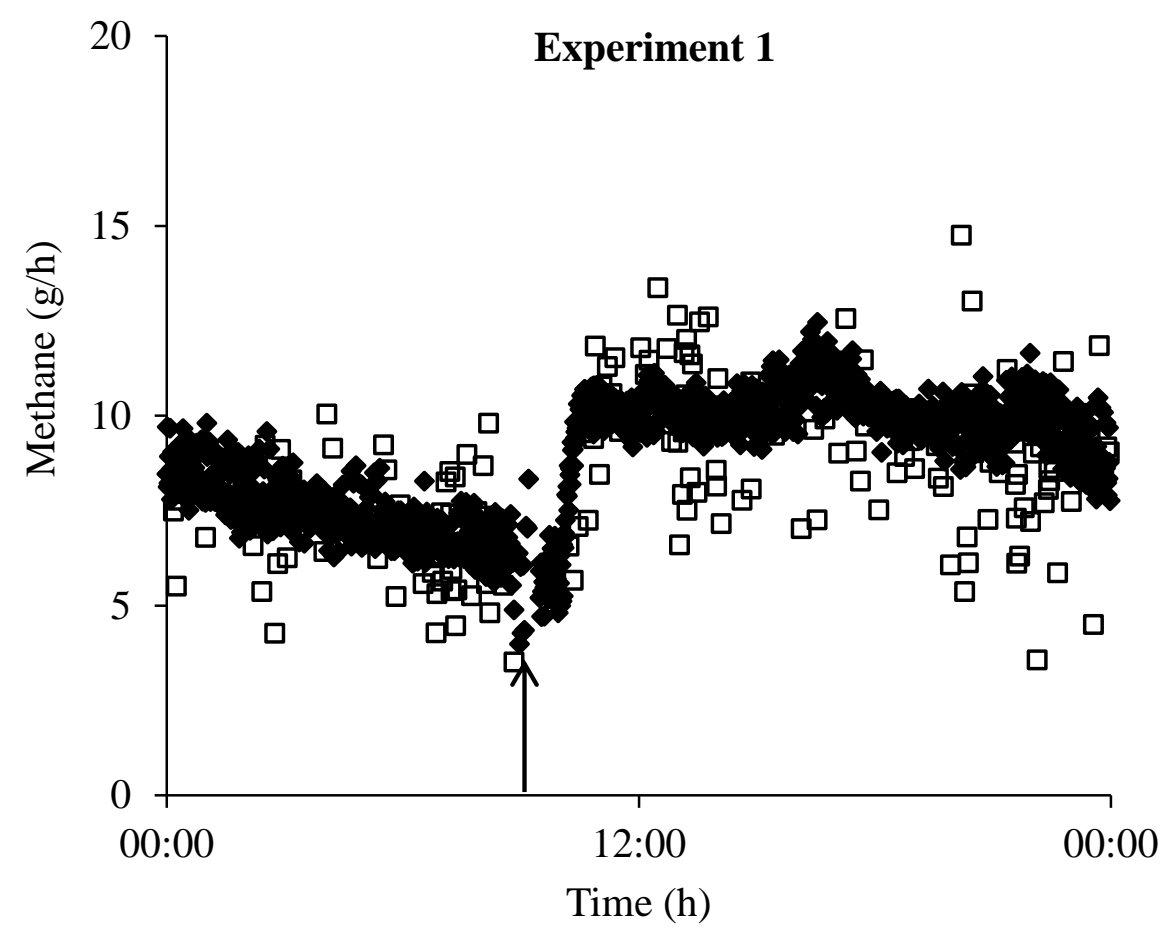

667

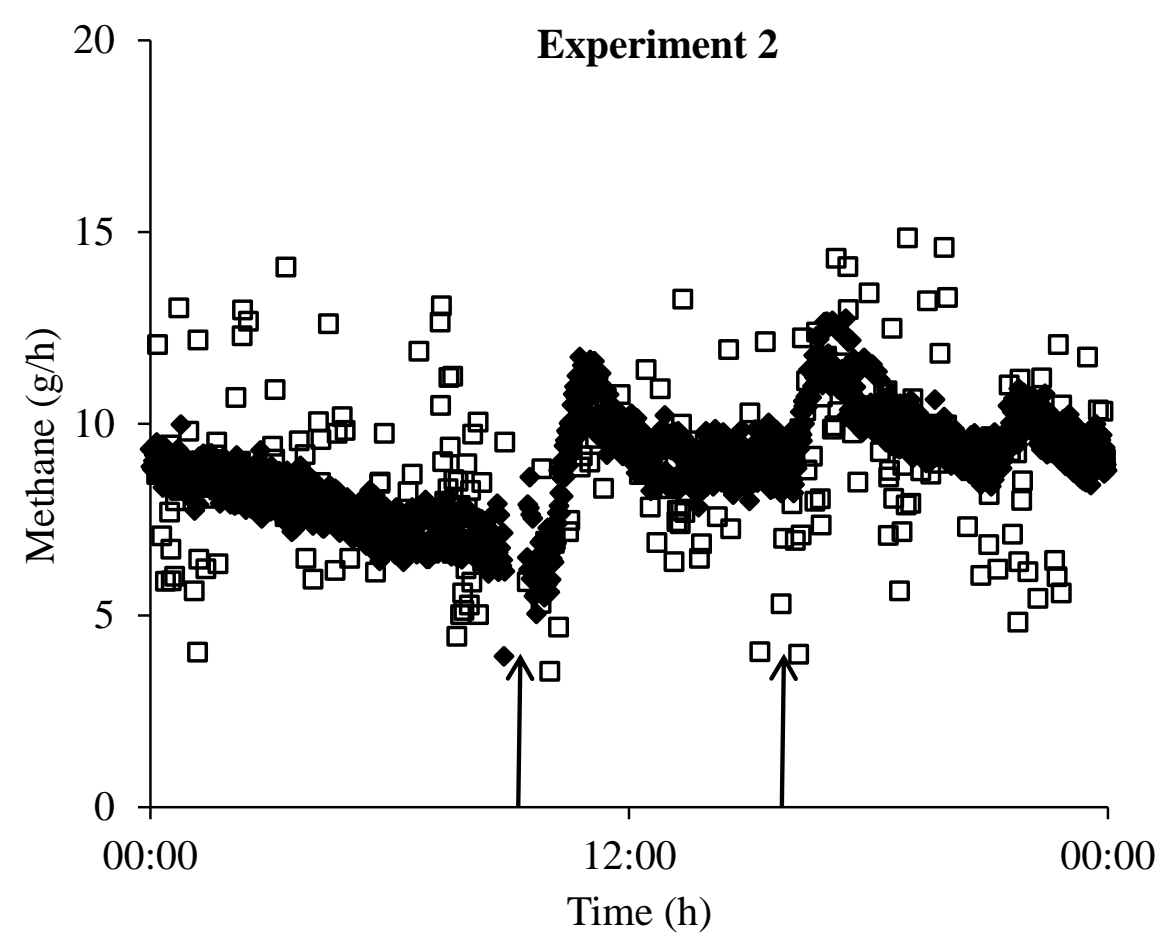

668

669 Fig 2. Comparison of methane emission rate ( $\mathrm{g} / \mathrm{h}$; minute average) measured using 670 GreenFeed (GF; open square symbol) and respiration chambers (RC; closed diamond 671 symbol) for all dairy heifers in experiments $1(n=8)$ and $2(n=16)$. There were $56 \mathrm{~d}$ GF and $67232 \mathrm{~d}$ RC measurements for experiment 1 , and $112 \mathrm{~d}$ GF and $64 \mathrm{~d} \mathrm{RC}$ for experiment 2. 673 Arrows indicate time of feeding. 


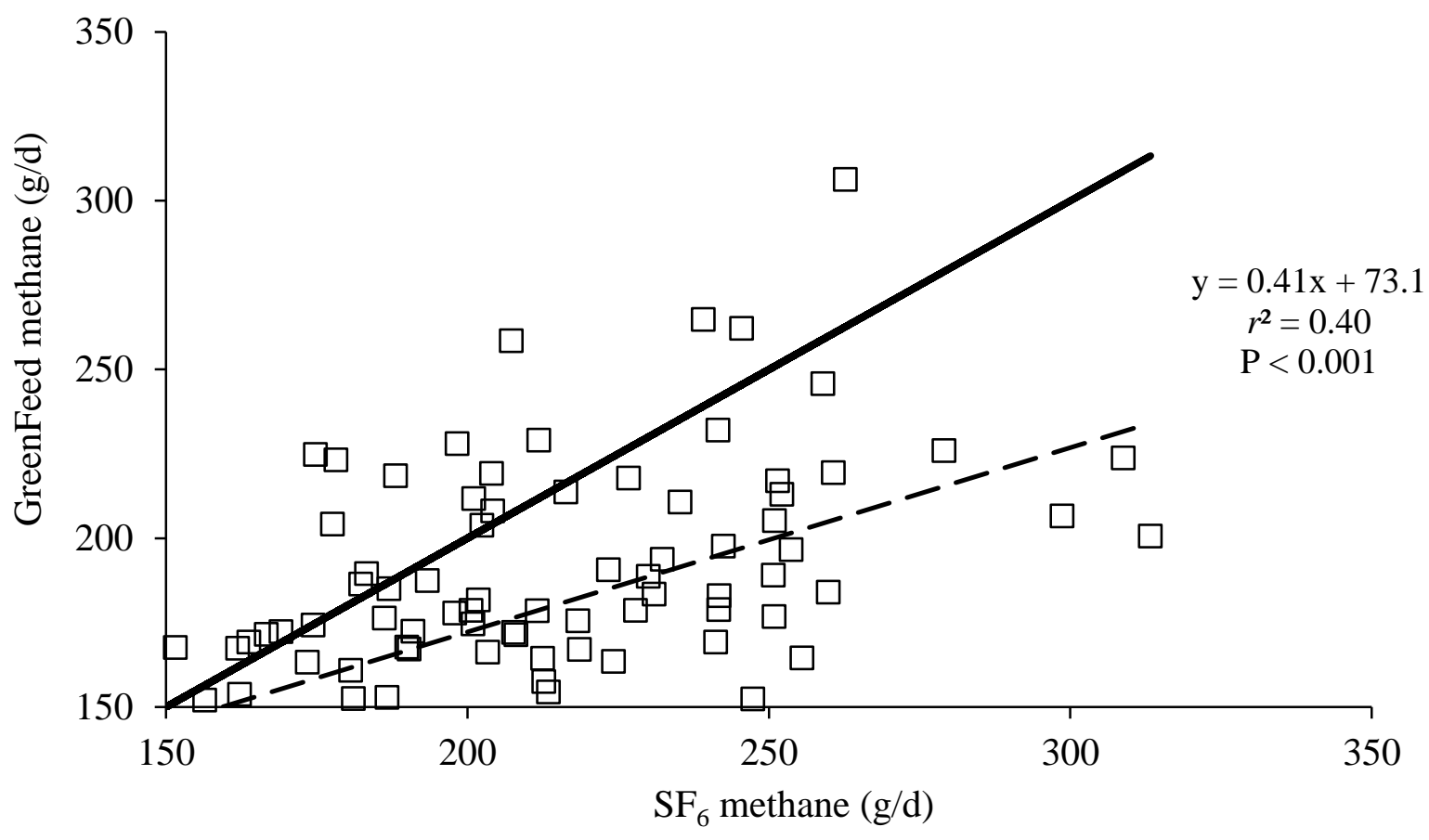

674

675 Fig 3. Relationship between methane production (g/d), determined using GreenFeed (GF)

676 and sulphur hexafluoride tracer $\left(\mathrm{SF}_{6}\right)$ techniques, of individual dairy heifers in experiment 3

$677(n=136)$. Solid line indicates $\mathrm{y}=\mathrm{x}$. Lin's Concordance value $=0.6017$. 


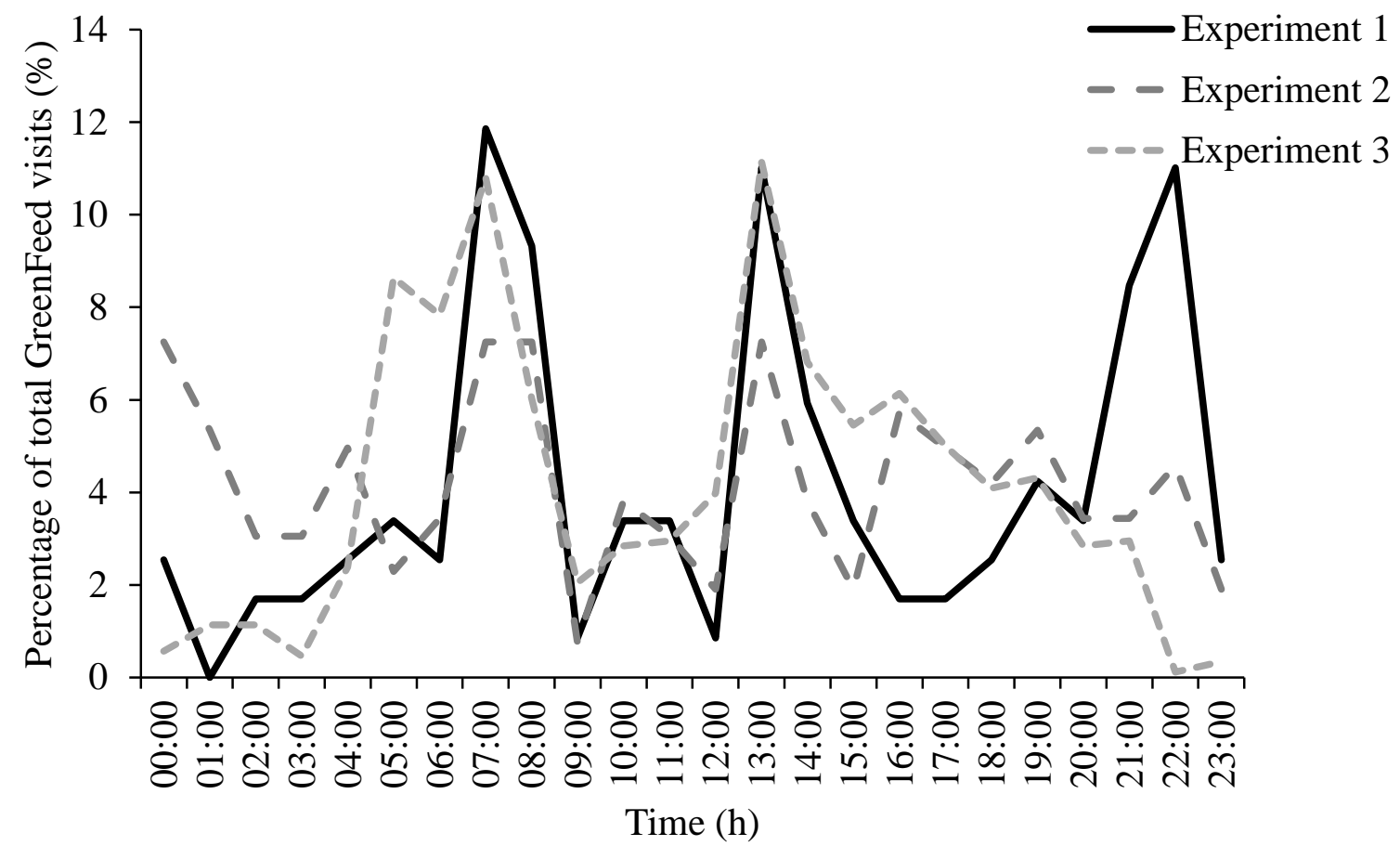

678

Time (h)

679 Fig 4. Diurnal pattern of GreenFeed (GF) visitation (methane measurements) over $24 \mathrm{~h}$, as a

680 percentage of total visits, by growing dairy cattle of experiments 1 (120 GF visits/14 d), 2

681 (262 GF visits/28 d) and 3 (880 GF visits/48 d). 